\title{
Cognitive aging: is there a dark side to environmental support?
}

\author{
Ulman Lindenberger ${ }^{1}$ and Ulrich Mayr ${ }^{2}$ \\ ${ }^{1}$ Center for Lifespan Psychology, Max Planck Institute for Human Development, Berlin, Germany \\ ${ }^{2}$ Department of Psychology, University of Oregon, Eugene, OR, USA
}

It has been known for some time that memory deficits among older adults increase when self-initiated processing is required and decrease when the environment provides task-appropriate cues. We propose that this observation is not confined to memory but can be subsumed under a more general developmental trend. In perception, learning or memory, and action management, older adults often rely more on external information than younger adults do, probably both as a direct reflection and indirect adaptation to difficulties in internally triggering and maintaining cognitive representations. This age-graded shift from internal towards environmental control is often associated with compromised performance. Cognitive aging research and the design of aging-friendly environments can benefit from paying closer attention to the developmental dynamics and implications of this shift.

\section{Environmental support in old age}

Thirty years ago, Fergus Craik [1] placed findings on adult age differences in memory on a continuum ranging from self-initiated processing to environmental support. According to Craik, memory performance is particularly impaired when retrieval depends on self-generated cues and active control processes. By contrast, when retrieval cues (e.g., hints, reminders, contextual reinstatement) are provided by the environment, age-related deficits decrease or disappear altogether (Box 1).

Craik explicated his claim by noting that self-initiated processing and constructive cue generation require a considerable amount of 'attentional resources', which decline with advancing adult age. From the current perspective, deploying attentional resources refers to maintaining task representations through recurrent connections between prefrontal and more posterior regions of the brain [2]. The ability to hold task representations in mind declines with age [3], as reflected by impairments in a variety of cognitive functions such as attention, working memory

Corresponding authors: Lindenberger, U. (lindenberger@mpib-berlin.mpg.de); Mayr, U. (mayr@uoregon.edu).

Keywords: environmental support; self-initiated processing; cognitive aging; cognitive control.

$1364-6613 / \$$ - see front matter

(c) 2013 Elsevier Ltd. All rights reserved. http://dx.doi.org/10.1016/j.tics.2013.10.006
(WM), and executive control $[4,5]$. Task-relevant cues serve as reminders of what to do and when, thereby reducing the need to trigger and maintain task representations internally. Hence, environmental support facilitates performance, especially in old age.

On the basis of these considerations, we may expect a gradual shift away from self-initiated processing towards reliance on environmental support with advancing adult age. This is indeed what we see: across processing stages and modalities, older adults are more likely to be guided by external cues than younger adults are. Here, we argue that this shift also comes at a cost, as the affordances of the environment increasingly dominate the structure and content of thought and behavior. To substantiate this claim, we scrutinize adult age differences in behavior from perception to goal-directed action. We examine developmental mechanisms underlying the tendency of older adults to outsource control to the environment, and discuss implications for environments that support successful aging.

\section{Perception: environmental entrainment}

Perceptual processing often requires that individuals detect infrequent events in the context of frequent distractor events. In an electroencephalograpy (EEG) experiment, Müller et al. asked younger and older adults to listen to a series of auditory stimuli consisting of a frequent and a rare ('oddball') tone [6]. Measures of phase locking and evoked power were used to quantify the extent to which perceptual processing was entrained by stimulus onset. In younger adults, individual differences in synchronization to attended stimuli were positively correlated with independently assessed measures of perceptual speed (Figure 1A). In older adults, however, individual differences in synchronization were negatively correlated with perceptual speed (Figure 1B). These results indicate that the early representation of auditory sensory events in old age occurs in a highly stimulus-driven manner that is less easily modulated by top-down influences and less easily integrated into ongoing cognitive activity.

Likewise, Passow et al. examined adult age differences in the interplay between perceptual saliency and attentional control over auditory processing [7] in a dichotic listening task originally introduced by Hugdahl et al. [8] and later refined by Westerhausen et al. [9]. Perceptual saliency was manipulated by decreasing the intensity of either the right- or the left-ear input in 5-dB steps until a maximum difference of $20 \mathrm{~dB}$ between ears was reached. 


\section{Box 1. Self-initiated processing versus environmental support: Craik's 1983 account}

Craik's article [1] marked a departure from the modal model of memory [59], which assumed that memory formation and retrieval are achieved as a series of transfers among separate places, or systems, for information storage, such as a short-term store and a long-term store. In line with the levels-of-processing approach [60], Craik proposed a functionalist approach to memory, or remembering, by taking a closer look at the kinds of cognitive activity people engage in during encoding and retrieval.

After having noted that both encoding and retrieval tasks vary in the degree to which they require self-initiated constructive operations, Craik illustrated the distinction between self-initiated processing and environmental support with the following examples (p. 112 [1]):

Some encoding operations are so well practiced, or the stimuli are so compatible with the relevant processing mechanisms that the encoding is carried out 'automatically,' without conscious effort (e.g. perceiving a picture or an expected word in context). Other encoding operations, for example, involving deductions or inference, require much more attention and effort. Retrieval tasks also vary in the degree to which they require self-initiated constructive operations; some, like free recall, involve minimal 'environmental support'.

At the other end of the continuum, Craik continued, we find 'procedural' tasks such as learning to read mirror-image script or solve jigsaw puzzles. These require relatively little self-initiated processing, because there is no need to go beyond the information provided by the environment to reconstruct details of the event, as in recall, or details of the original context of occurrence, as in recognition.

On the basis of this line of reasoning, Craik arranged experimental paradigms for memory retrieval in an order that is supposed to reflect the degree to which retrieval operations are either driven by the environment or by self-initiated activities (Figure I). On the basis of assumptions that normal aging depletes attentional resources and

The 0-dB difference condition served as the baseline intensity and was adapted to each participant's individual hearing threshold. Twelve different dichotic syllable pairs were presented twice for each of the nine interaural intensity conditions. Attentional focus was manipulated by instructing the participants to focus on the right ear, on the left ear, or on both ears (neutral focus). When stimulus of the attended ear is louder, then attention is facilitated by saliency; however, when stimulus of the attended ear is softer, then attention has to overcome the saliency advantage of stimuli presented to the unattended ear. Across all interaural intensity conditions, younger adults were capable of flexibly focusing their attention on auditory inputs from either the right or left ear (Figure 2A). In stark contrast to younger adults, the performance of older adults was driven almost exclusively by perceptual saliency, with attentional focus having little effect on performance (Figure 2B). In a follow-up event-related potential (ERP) study, Passow et al. observed a late negativity at frontocentral and parietal electrodes in younger adults that was sensitive to perceptual-attentional conflicts and correlated positively with task performance [10]. In older adults, this component was absent.

The findings reported thus far show that auditory stimuli entrain the cognitive systems of older adults in relatively mandatory ways that are not easily modulated or overcome by attentional control. In the visual domain, the picture is similar. Using a cued change detection task with hemifield presentation introduced by Vogel et al. [11], several studies have found that older adults are more likely that resource demands increase monotonically with the amount of self-initiated activity, he hypothesized that the magnitude of agerelated performance impairments should increase with the need for self-initiated processing. The results of a meta-analysis by La Voie and Light [61] are in good agreement with this hypothesis (Figure l; cf. [62]): the effect sizes of the performance advantage of younger over older adults are large for free recall, moderate for recognition memory, and small for associative and item priming (i.e., paradigms taxing procedural learning).
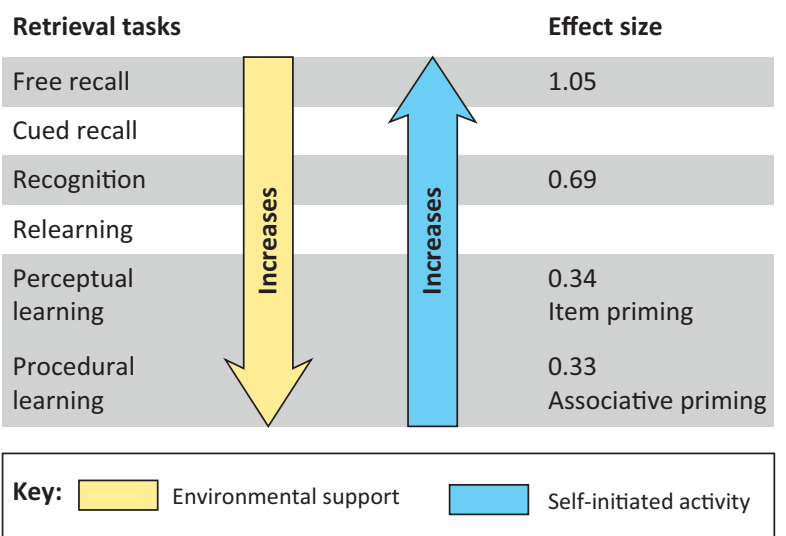

$\overline{T R E N D S}$ in Cognitive Sciences

Figure I. A hypothetical rank order of the relative importance of environmental support and self-initiated processing for different experimental paradigms of learning and memory. The numbers to the right of the figure are based on a meta-analysis by La Voie and Light [61]. They show effect size estimates for the performance advantage of younger over older adults. The data follow the hypothesized order [62]. Adapted from Craik [1] with permission.

to encode and process distractors than younger adults are $[12,13]$. Following up on a paradigm introduced by Vogel and colleagues, Sauseng et al. found that the amplitude of alpha oscillations during the retention interval is greater ipsilaterally than contralaterally to the attended hemifield [14]. The authors also noted that the lateralized difference in alpha power increased with load and predicted individual WM capacity, confirming that alpha oscillations play a role in inhibitory processing $[15,16]$. On the basis of these findings, Sander et al. compared inhibitory control over WM contents in children, younger adults, and older adults [17]. To keep task difficulty comparable across age groups, children and older adults worked under easier load conditions than younger adults did. Ipsilateral alpha oscillations were weaker in older adults than in younger adults and children, in line with less efficient inhibitory control among older adults. At the same time, older adult showed greater phase locking of alpha oscillations immediately after stimulus presentation than both younger adults and children did. These results agree with those in the auditory domain, and again suggest that high levels of phase locking among older individuals may reflect mandatory stimulus processing that is not easily modulated by task requirements.

Other studies with visual stimuli have confirmed that older adults have difficulties in resisting indiscriminate entrainment by distractors, disengaging from distracting information, or both [12,18-25] (for general accounts, see $[4,5,26])$. For instance, Störmer et al. found that normal aging compromises early multifocal visual attention 


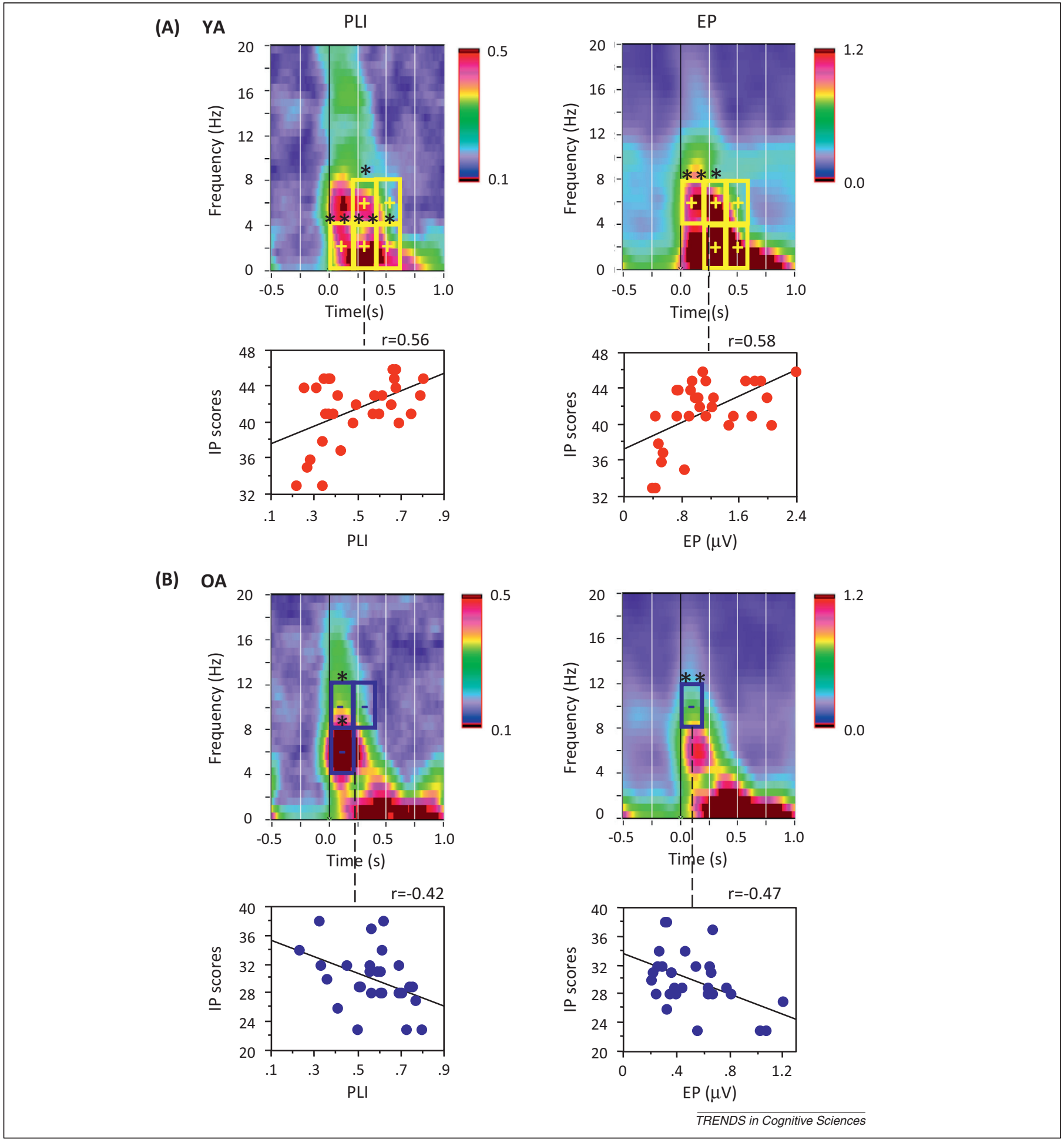

Figure 1. Adult age differences in oscillatory stimulus entrainment during auditory oddball performance. ( $A, B)$ Younger and older adults listened to a series of auditory stimuli consisting of a frequent and a rare, deviant tone (the 'oddball'). The phase locking index (PLI, [63]) and evoked power (EP) were used to quantify the extent to which perceptual processing was entrained by the stimulus. The heat maps display grand average stimulus-locked time-frequency maps for PLI and EP under the central electrode $\mathrm{Cz}$ when attending to the deviant stimulus. In each heat map, a vertical line marks the stimulus onset and rectangles mark the time-frequency ranges used to compute average PLI or EP values. Yellow rectangles with a plus sign indicate statistically significant positive correlations, and blue rectangles with a minus sign indicate statistically significant negative correlations with Identical Pictures (IP), an independently assessed test of perceptual speed. Scatter plots illustrating select correlations are shown below each heat map. (A) In younger adults (YA), greater phase locking and higher EP are positively related to perceptual speed. (B) In older adults (OA), greater phase locking and higher EP are negatively related to perceptual speed. The data suggest that the early representation of auditory sensory events in old age occurs in a highly stimulus-driven manner that is less easily modulated by top-down influences. This interpretation is strengthened by additional data (not shown here) indicating that the amount of stimulus-induced synchronization as assessed by PLI and EP varies less as a function of stimulus type (deviant vs standard) and instruction in older adults than in younger adults. Adapted from Müller et al. [6] and reprinted with permission. 


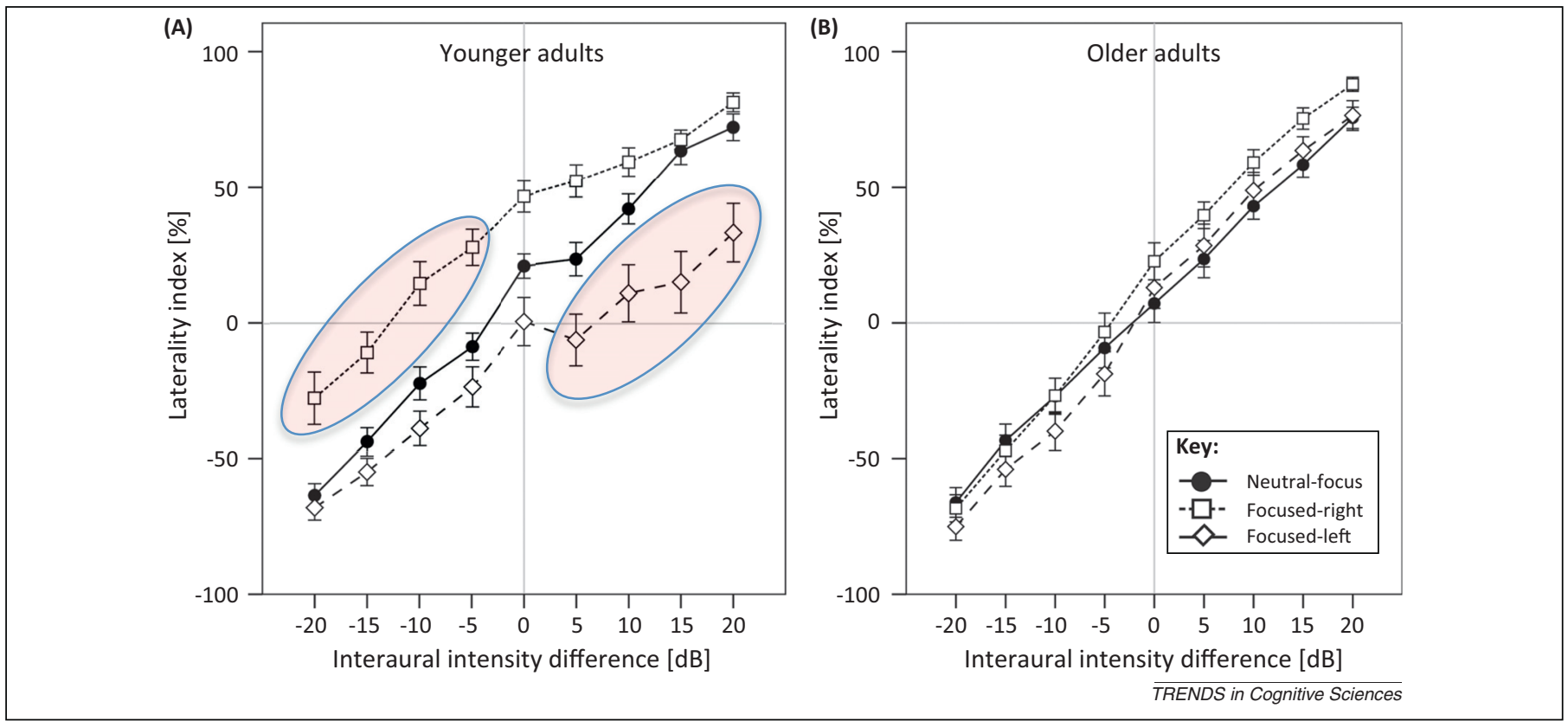

Figure 2. Adult age differences in auditory attentional control. Participants were presented with dichotic pairs of voiced versus unvoiced syllables (e.g., /ba/ versus /pa/) and were asked to report the syllable heard. Perceptual saliency, shown on the $x$-axis, was manipulated by decreasing the intensity of either the right- or the left-ear input in 5 -dB steps until a maximum difference of $20 \mathrm{~dB}$ between ears was reached. Negative values represent conditions in which left-ear stimuli were louder than right-ear stimuli, and

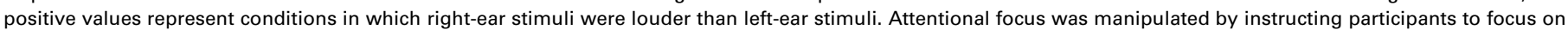
the right ear, on the left ear, or on both ears (neutral focus). Reports are quantified by the laterality index, shown on the $y$-axis, which expresses the amount of right-ear reports in relation to left-ear reports \{i.e., [(right ear - left ear) / (right ear + left ear)] $\times 100\}$. The laterality index ranges from $-100 \%$ to $+100 \%$, with positive values indicating a right-ear and negative values a left-ear advantage. When the stimulus for the attended ear is louder, then attention is facilitated by saliency; when the stimulus for the attended ear is softer, then the saliency advantage of the stimuli presented to the unattended ear has to be overcome by top-down attentional control. In contrast to (A) younger adults, who were capable of flexibly focusing their attention on auditory inputs from either the right or left ear, performance in (B) older adults was driven almost exclusively by perceptual saliency. In particular, the distance between the data highlighted in pink and the data point from the neutral-focus condition underscore the ability of younger adults to use top-down modulation to overcome conflicts between perceptual saliency and attentional focus; the overlap between the corresponding conditions among older adults indicates that this ability is severely impaired in old age. Figure adapted from Passow et al. [7] with permission.

during object tracking, as indicated by reduced attentional modulation of the P1 component of the ERP [24]. Using a response conflict task, Hämmerer et al. found that older adults showed a larger P3a component elicited by infrequent non-cue stimuli than children, adolescents, and younger adults did [20].

Finally, evidence from two-choice decision tasks shows that older adults adopt decision boundaries that are wider and farther away from the optimal speed-accuracy tradeoff than the boundaries of younger adults $[27,28]$. This can be seen as a greater reliance on environmental support. It also is consistent with the longstanding observation that older adults are more cautious in the sense that they prefer to take in more information before committing themselves to action [29-31].

\section{Action: environmental outsourcing}

Self-initiated cognitive control of goal-directed action is typically attributed to abstract representations of the current task set, again aided by prefrontal activity [2]. This activity can be changed on the fly when task sets need to be changed [32]. High-level task set representations constrain the low-level associative machinery that enables automatic selection of thought and action. In turn, these representations can also become part of associative processes, which then constrain the selection of task set representations. These conclusions are based on evidence indicating that task-set representations are bound to specific stimulus properties over a wide range of timescales [33,34]. For example, Mayr and Bryck asked participants to switch between two tasks by touching response fields located within one of two objects on a touch screen [33]. Taskswitch interference effects were much reduced when each task was consistently mapped to an object location, relative to a control situation in which both tasks were mapped to the same object; for similar findings, see [35,36].

Stimulus-to-task-set bindings provide a powerful mechanism for implementing environmental support. To the degree to which control demands are consistently mapped to certain aspects in the environment, these bindings automatically activate the necessary control representations. Cognitive control no longer relies exclusively on internally triggered and maintained representations, but instead can be 'outsourced' to the environment.

As we might expect, low-level perceptual affordances constrain high-level task representations to a greater extent in older than in younger adults. For instance, Mayr found that the negative influence of low-level stimulusresponse repetitions on task-switch costs is particularly large among older adults [37]. In addition, older adults show larger global or mixing costs [38,39] than younger adults do, that is, slowed responses to no-switch trials that occur in the context of task-switch blocks. Global switching costs are assumed to originate from involuntary, stimulusdriven activation of the currently irrelevant task set [40]. In sum, the greater reliance of older adults on external stimuli compromises their ability to implement one among several competing task sets. 
A particularly striking example of the costs of relying on the environment to support control processes comes from a variant of the task-switching paradigm, the so-called fadeout procedure (Box 2). Here, older adults show marked difficulties in transitioning mid-block from an initial phase of trial-to-trial task switching to a second phase of exclusive focus on a single task (i.e., the fade-out phase). By contrast, younger adults have almost no difficulties with this transition. Monitoring of eye movements during the fade-out phase reveals that older adults continue to fixate on the on-screen task cues in over $80 \%$ of the trials, compared to only $15-20 \%$ for young adults. The binding between task cues and task selection forces older adults into a control mode that was useful for the initial taskswitching phase, but is no longer needed during the fadeout phase.

The over-reliance of older adults on external information also becomes apparent in an experimental situation that participants can solve either by retrieving relevant information about word pairs from memory or by simply inspecting the information from a look-up table provided on the screen [41]. Retrieval is faster than visual scanning of the table, and with some practice, younger adults quickly transition to retrieval. By contrast, for older adults, timeconsuming scanning remains the dominant strategy, considerably slowing down their performance.

In sum, we propose that older adults are more likely than younger adults to disrupt goal-directed activity by stimulus-driven inspections of the environment that delay, sidetrack, or even block fluent goal pursuit.

\section{Developmental mechanisms promoting environmental control in old age}

So far, we have shown that normal aging is associated with increased reliance on environmental support, not only in relation to memory, as originally stated by Craik [1], but

\section{Box 2. The fade-out paradigm}

The fade-out paradigm assesses the ability of individuals to transition from an initial high-internal control phase that requires trial-by-trial selection of relevant task sets to a situation in which only one task remains relevant and no task selection is necessary. Figure IA shows the basic conditions and their block structure. In the experimental condition (fade-out condition), participants begin by switching on a trial-by-trial basis between two tasks (judging either the color or shape of an object) based on the visual task cue (black circle under the verbal task label indicates the relevant task). From a certain trial onwards (in this example, trial 44), one of the two tasks is faded out. The corresponding task label is crossed out and does not become relevant again for the rest of the block. In addition, prior to each block, participants are painstakingly instructed about the structure of the upcoming block. This experimental condition is compared to a control condition in which a single task is relevant throughout the entire block. Fade-out RT costs are computed by subtracting RTs from the fade-out phase from the corresponding RTs for the single-task control condition. Figure IB shows the typical pattern of age differences in fade-out costs (labeled 'With cues'). Whereas younger adults transition very quickly to a single-task performance level (fade-out costs approach zero), older adults exhibit large costs that do not disappear for the entire block. Figure IB also shows results for a 'No cue' condition in which the visual task labels for the fade-out phase are eliminated from the screen. Here, age differences in fade-out costs are dramatically reduced (for further discussion, see the section on developmental mechanisms).
(A)
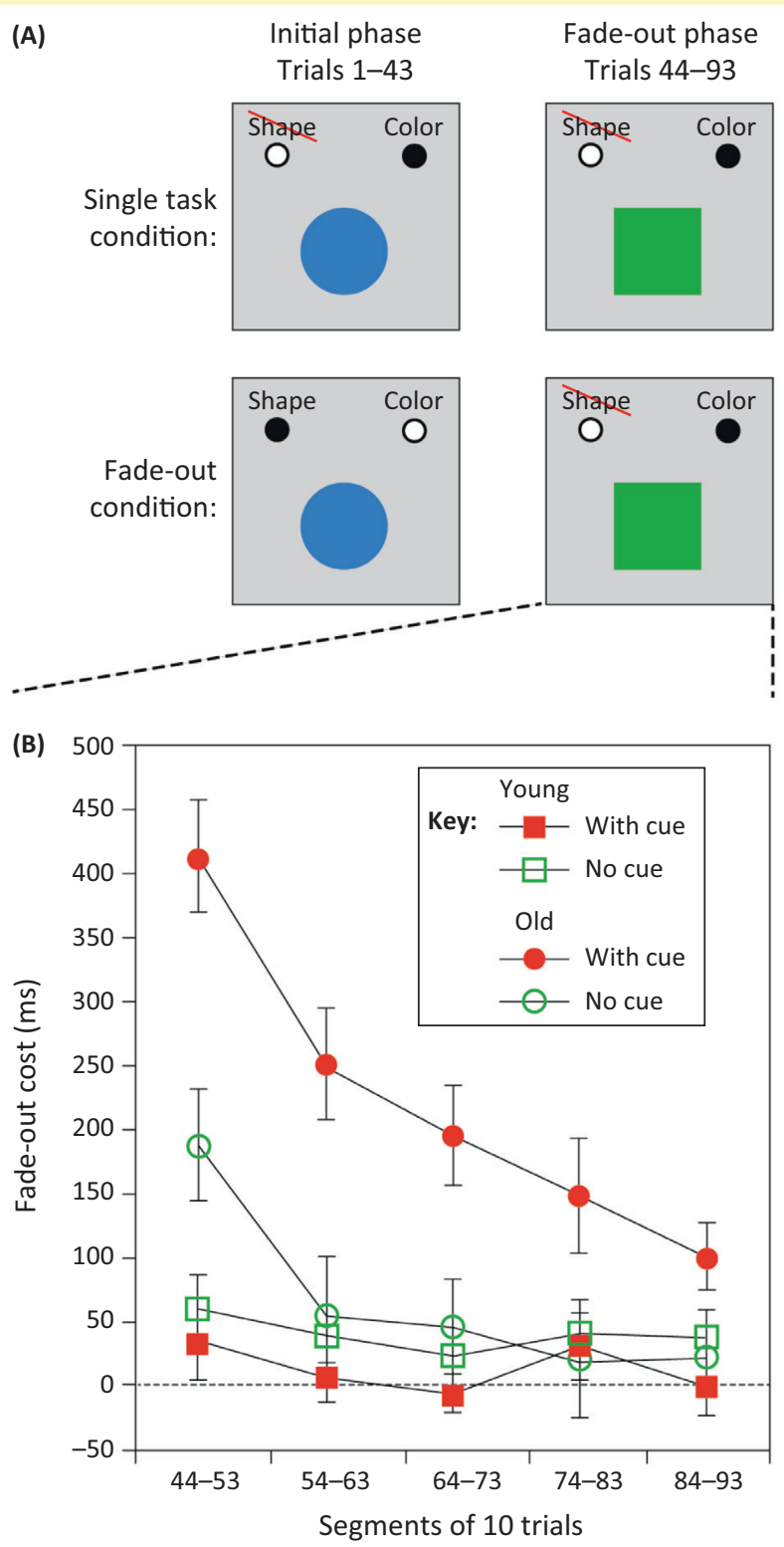

$\overline{\text { TRENDS in Cognitive Sciences }}$

Figure I. (A) The fade-out paradigm. (B) Results from Spieler et al. [50]. Reprinted with permission.

also in relation to perceptual processing and goal-directed action. In our view, theories about cognitive aging need to acknowledge the generality of this shift and search for potential explanations. In this regard, the very fact that, despite clear costs (Figures 1 and 2), older adults show greater environmental entrainment than younger adults do is revealing. This likely rules out the possibility that the greater orientation towards environmental cues results from an explicit choice that relies on an analysis of immediate costs and gains [42].

Future research needs to address the reasons for the greater reliance of older adults on the environment. For example, does loss of internal control due to compromised prefrontal functions [3,4] come first, making external control necessary, or do older adults sometimes choose external control even though they would be able to adopt internal control strategies? In our view, there is evidence 
of both. The first explanation follows directly from the framework of biased competition [43], according to which the responses of the brain to any particular problem reflect the outcome of mutual competition between bottom-up perceptual and motor representations on the one hand, and top-down biasing signals representing more abstract task-set and context representations on the other. From this perspective, impairments in the strength and reliability of top-down biasing directly lead to a greater contribution of bottom-up representations to behavior (Figure 3A). We term this explanation the direct-link account because the increase in environment control is directly coupled to the declining fidelity and strength of top-down control. The age-comparative pattern shown in Figure 2 matches this scenario perfectly: when top-down control is impaired, perceptual saliency takes over.

In general, a broad array of findings on perceptual processing and interference in WM seems to support the notion of a direct link between waning cognitive control and increasing domination by environmental cues; for summaries, see Gazzaley [4] and Sander et al. [44]. From this perspective, it might even be argued that there is not much new to our account: the greater reliance on environmental support is just a side-effect of weakened internal control representations, along with other consequences, such as the well-documented tendency of older adults to rely on over-learned memory scripts and schematic information rather than on an effortful analysis of the current situation [45].

We believe, however, that the direct-link account, while plausible and parsimonious, needs to be complemented by an additional layer that allows for a more indirect connection between waning internal cognitive control and increasing reliance on environmental support. If greater reliance on the environment were always a direct reflection of weakened internal representations, we would expect the performance of older adults to suffer promptly when environmental support is eliminated. This is not always true removal of environmental sources of information may sometimes even improve the task performance of older adults. For example, when task cues are absent during the final, single-task phase of the fade-out paradigm, the accuracies of older adults remain very high - which defies the notion of weakened internal representations - and their fade-out costs are substantially reduced (Box 2). Similarly, in experiments conducted by Hertzog and colleagues, well-trained older adults were able to switch to a retrieval strategy once the look-up table was removed, even though they preferred to use the look-up table when it was present [46]. In both cases, older adults rely on the environment even though their internal representations are fully capable of handling current task demands fluently and accurately. These findings are inconsistent with the notion that increasing environmental control is always the direct mirror image of waning internal control.

Instead, we suggest that the greater reliance of older adults on the environment may also reflect a long-term adaptation to a cognitive system that in general - but not necessarily in every specific instance - is less capable of directing behavior in a top-down, internally regulated manner $[47,48]$. We call this the indirect-link account

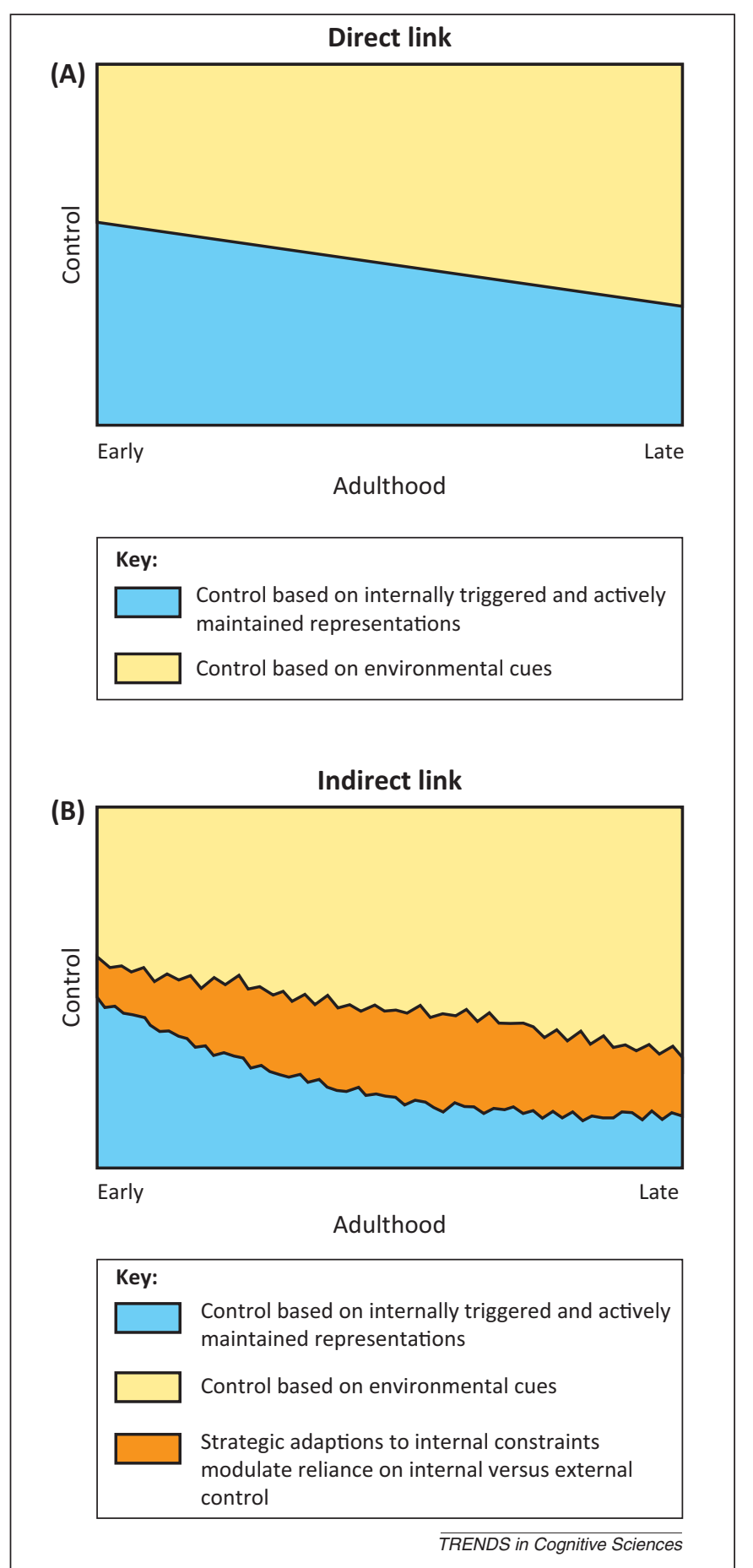

Figure 3. Two ways in which reliance on actively maintained representations and external support may change from early to late adulthood. (A) Direct link: reliance on external support is inversely linked to the robustness of top-down, internally maintained representations. (B) Indirect link: reliance on external support also depends on strategic adaptations to attenuate the adverse consequences of less robust internal representations. Hence, the same amount of decline in top-down control can lead to different developmental trajectories of environmental support across individuals, and within individuals across different domains.

(Figure 3B). It posits a certain amount of slack between deficits in self-initiated processing and reliance on environmental support, and predicts that individuals are capable, in some situations at least, of reducing their degree of reliance on the environment when they need to do so. Accordingly, Touron, Hertzog, and colleagues have shown that older adults are more likely to switch to a retrieval 
strategy when the costs of visual scanning or the benefits of retrieval are increased [46,49].

Direct and indirect pathways from the waning of topdown control to a greater reliance on environmental support likely co-exist, and little is known about their relative importance and developmental interdependence. In-depth analyses of experimental data were needed to identify the indirect nature of fade-out costs [50] and the reluctance of older adults to rely on an existing, episodic, long-term memory base [46]. The jury is out on the degree to which other empirical phenomena that are typically viewed as direct results of weakened WM or inhibitory resources instead fall into the indirect link category. To assess the relative explanatory power of direct- and indirect-link processes, the association between the tendency to rely on the environment and the quality of internal representations needs to be examined across different paradigms and individuals. Importantly, internal and external control

\section{Box 3. New forms of environmental support: adaptive assistive technology}

If environmental cues increasingly control behavior with advancing age, then their properties should attract the attention of cognitive aging researchers [64-66]. Two such properties, compatibility and distinctiveness, are noteworthy $[67,68]$. Cues are compatible when they point to attributes that are functionally related to the taskrelevant memory episode or action tendency, and distinctive when they activate the target action without co-activating a large number of competing actions.

Individuals often generate their own cues, either explicitly or implicitly as sediments of their earlier actions. Such cues are likely to match their knowledge, habits, and preferences, and should show superior compatibility and distinctiveness. A study by Mäntylä confirmed this expectation [67]. In a cued-recall task, Mäntylä asked college students during the study phase to define their own retrieval cues by generating properties or features for each word to be remembered. When presented as cues during recall, self-generated properties resulted in exceptionally high levels of memory performance. For example, after 7 days, participants recalled on average 327 of the $\mathbf{5 0 4}$ words when given three self-generated properties as cues; immediately after study, they recalled 459 of the 504 words. Mäntylä also found that self-generated retrieval cues were far more effective than cues generated by another person. Similar, although somewhat attenuated effects have been observed among older adults [69].

Adaptive technology can assist aging individuals by providing cuing structures that connect properties in the environment to their action goals $[64,65,70]$. To this end, the assistive device (e.g., smartphone) or the instrumented environment (e.g., smart kitchen) needs to learn the user's habits and preferences [71]. Initially, when knowledge about the individual user is absent, the device may operate with default settings that are based on a model of the 'average user'. Explicit off-line information about the user's cognitive, sensory, and sensorimotor abilities, as well as his or her preferences and habits, may be added to modify these default parameters. This would be followed by an extended period of person-specific acculturation of the device, in the course of which the device learns the regularities and contingencies that permeate the life of the individual user, and provides prompts to assist users in pursuing their goals if needed.

When gauging the long-term consequences of adaptive assistive technology, risks and opportunities need to be kept in mind $[64,65]$. Chronic reliance on technological aids may deplete brain resources through protracted disuse of skills and abilities, undermine motivation, and engender loss of autonomy. However, adaptive assistive technology may foster cognitive maintenance and plasticity [72-74] by combining support with challenge, thereby enhancing motivation [75], social participation, and a sense of autonomy, with positive repercussions on cognitive development in old age [76].

\section{Box 4. Outstanding questions}

- Which neural changes trigger and sustain the increasing reliance of older adults on environmental cues?

- Is the increasing reliance on environmental support with advancing adult age a domain-general change, or do its trajectories and mechanisms differ across perception, memory, and action control?

- What are the relative importance and dynamics of direct and indirect pathways towards greater environmental control? How do these pathways differ across individuals? Why are some older individuals better able to maintain high levels of internal control in old age than others are?

- Which properties of the environment strengthen the bright and weaken the dark side of environmental support?

modes need to be tracked over time within the same individuals to elucidate the longitudinal dynamics between the two pathways and the range of individual differences therein [51]. Moreover, the tendency to outsource control to the environment has also been observed in other situations of compromised top-down control, such as fatigue and sleepiness [52]. It seems instructive to compare these states to the effects of normal cognitive aging.

\section{Environments for successful aging}

In this article, we revisited Craik's 1983 observation that adult age differences are attenuated when processing is supported by task-relevant external cues, and amplified when processing has to be initiated and maintained in the absence of such cues [1]. This observation has received ample empirical support beyond the functional domain of memory for which it was originally conceived. Relative to younger adults, the cognitive systems of older adults are entrained by perceptual stimuli in ways that are less a reflection of attentional focus, and more a reflection of perceptual salience. In addition, when the environment provides prompts for action, older adults are more likely than younger adults to rely on these prompts, even when they do not need them and even when they hamper fluent performance.

Environmental support has a bright and a dark side: it helps aging individuals to perform but comes with a loss in internal control. It follows that the environment matters, especially in old age. The initiation and maintenance of internal control are costly, both cognitively $[42,53]$ and metabolically [54], and these costs appear to increase from early to late adulthood [55-57]. By the time they have reached old age, individuals have acquired a behavioral repertoire that is likely to match the regularities and affordances of the environments they live in. The tendency of older adults, both automatic and deliberate, to outsource control to the environment may be inefficient at times, but cost-effective in the long run if the cuing structure of the environment corresponds to their goals and needs. Engineers, psychologists, and aging individuals themselves need to keep this in mind as they design and use adaptive technology in the pursuit of successful aging (Box 3) [58].

Box 4 summarizes outstanding questions.

\section{Acknowledgments}

U.L. would like to thank the Max Planck Society for continued research support. Parts of the research described in this article were financed by a 
Gottfried Wilhelm Leibniz Award 2010 of the DFG to U.L. and an NIA grant (R01 AG037564-01A1) to U.M. We thank Gus Craik, Kenneth Hugdahl, and Susanne Passow for comments on earlier versions of this article.

\section{References}

1 Craik, F.I.M. (1983) On the transfer of information from temporary to permanent memory. Philos. Trans. R. Soc. Lond. B: Biol. Sci. 302, 341359

2 Ardid, S. et al. (2007) An integrated microcircuit model of attentional processing in the neocortex. J. Neurosci. 27, 8486-8495

3 Wang, M. et al. (2011) Neuronal basis of age-related working memory decline. Nature 476, 210-213

4 Gazzaley, A. (2013) Top-down modulation deficit in the aging brain. An emerging theory of cognitive aging. In Principles of Frontal Lobe Function (2nd ed.) (Stuss, D.T. and Knight, R.T., eds), pp. 593-608, Oxford University Press

5 Hasher, L. and Zacks, R.T. (1988) Working memory, comprehension, and aging: a review and a new view. Psychol. Learn. Motiv. 22, 193-225

6 Müller, V. et al. (2009) Lifespan differences in cortical dynamics of auditory perception. Dev. Sci. 12, 839-853

7 Passow, S. et al. (2012) Human aging compromises attentional control of auditory perception. Psychol. Aging 27, 99-105

8 Hugdahl, K. et al. (2008) The effect of stimulus intensity on the right ear advantage in dichotic listening. Neurosci. Lett. 431, 90-94

9 Westerhausen, R. et al. (2009) Top-down and bottom-up interaction: manipulating the dichotic listening ear advantage. Brain Res. 1250 183-189

10 Passow, S. et al. (2012) Electrophysiological correlates of adult age differences in attentional control of auditory processing. Cereb. Cortex http://dx.doi.org/10.1093/cercor/bhs306

11 Vogel, E.K. et al. (2005) Neural measures reveal individual differences in controlling access to working memory. Nature 438, 500-503

12 Jost, K. et al. (2011) Are old adults just like low working memory young adults? Filtering efficiency and age differences in visual working memory. Cereb. Cortex 21, 1147-1154

13 Sander, M.C. et al. (2011) Contralateral delay activity reveals lifespan age differences in top-down modulation of working memory contents. Cereb. Cortex 21, 2809-2819

14 Sauseng, P. et al. (2009) Brain oscillatory substrates of visual shortterm memory capacity. Curr. Biol. 19, 1846-1852

15 Kelly, S.P. et al. (2006) Increases in alpha oscillatory power reflect an active retinotopic mechanism for distracter suppression during sustained visuospatial attention. J. Neurphysiol. 95, 3844-3851

16 Rihs, T.A. et al. (2007) Mechanisms of selective inhibition in visual spatial attention are indexed by alpha-band EEG synchronization. Eur. J. Neurosci. 25, 603-610

17 Sander, M.C. et al. (2012) Amplitude modulations and inter-trial phase stability of alpha-oscillations differentially reflect working memory constraints across the lifespan. Neuroimage 59, 646-654

18 Cashdollar, N. et al. (2012) Prolonged disengagement from attentional capture in normal aging. Psychol. Aging 28, 77-86

19 Gazzaley, A. et al. (2008) Age-related top-down suppression deficit in the early stages of cortical visual memory processing. Proc. Natl. Acad. Sci. U.S.A. 105, 13122-13126

20 Hämmerer, D. et al. (2010) An electrophysiological study of response conflict processing across the lifespan: assessing the roles of conflict monitoring, cue utilization, response anticipation, and response suppression. Neuropsychologia 48, 3305-3316

21 McCarley, J.S. et al. (2012) Age, clutter, and competitive selection. Psychol. Aging 27, 616-626

22 Schmitz, T.W. et al. (2010) Failing to ignore: paradoxical neural effects of perceptual load on early attentional selection in normal aging. $J$. Neurosci. 30, 14750-14758

23 Störmer, V.S. et al. (2013) Normative shifts of cortical mechanisms of encoding contribute to adult age differences in visual-spatial working memory. Neuroimage 73, 167-175

24 Störmer, V.S. et al. (2013) Normal aging delays and compromises early multifocal visual attention during object tracking. J. Cogn. Neurosci. $25,188-202$

25 Zanto, T.P. et al. (2010) Predictive knowledge of stimulus relevance does not influence top-down suppression of irrelevant information in older adults. Cortex 46, 564-574
26 Hasher, L. et al. (1999) Inhibitory control, circadian arousal, and age. In Attention and Performance XVII (Gopher, D. and Korint, A., eds), pp. 653-675, MIT Press

27 Starns, J.J. and Ratcliff, R. (2010) The effects of aging on the speedaccuracy compromise: boundary optimality in the diffusion model. Psychol. Aging 25, 377-390

28 Starns, J.J. and Ratcliff, R. (2012) Age-related differences in diffusion model boundary optimality with both trial-limited and time-limited tasks. Psychon. Bull. Rev. 19, 139-145

29 Botwinick, J. et al. (1958) The interaction effects of perceptual difficulty and stimulus exposure time on age differences in speed and accuracy of response. Gerontologia 2, 1-10

30 Rabbitt, P. and Birren, J.E. (1967) Age and responses to sequences of repetitive and interruptive signals. J. Gerontol. 22, 143-150

31 Welford, A.T. (1961) Age changes in the times taken by choice, discrimination and the control of movement. Gerontologia 5, 129-145

32 Miller, E.K. and Cohen, J.D. (2001) An integrative theory of prefrontal cortex function. Annu. Rev. Neurosci. 24, 167-202

33 Mayr, U. and Bryck, R.L. (2005) Sticky rules: integration between abstract rules and specific actions. J. Exp. Psychol. Learn. Mem. Cogn. $31,337-350$

34 Waszak, F. et al. (2003) Task-switching and long-term priming: role of episodic stimulus-task bindings in task-shift costs. Cogn. Psychol. 46, 361-413

35 Crump, M.J.C.et al. (2006) The context-specific proportion congruent Stroop effect: location as a contextual cue. Psychon. Bull. Rev. 13, 316321

36 Mayr, U. and Bryck, R.L. (2007) Outsourcing control to the environment: effects of stimulus/response locations on task selection. Psychol. Res. 71, 107-116

37 Mayr, U. (2001) Age differences in the selection of mental sets: the role of inhibition, stimulus ambiguity, and response-set overlap. Psychol. Aging 16, 96-109

38 Verhaeghen, P. et al. (2003) Aging and dual-task performance: a metaanalysis. Psychol. Aging 18, 443-460

39 Kray, J. and Lindenberger, U. (2000) Adult age differences in task switching. Psychol. Aging 15, 126-147

40 Rubin, O. and Meiran, N. (2005) On the origins of the task mixing cost in the cuing task-switching paradigm. J. Exp. Psychol. Learn. Mem. Cogn. 31, 1477-1491

41 Rogers, W.A. et al. (2000) An individual differences analysis of ability and strategy influences: age-related differences in associative learning. J. Exp. Psychol. Learn. Mem. Cogn. 26, 359-394

42 Botvinick, M.M. et al. (2004) Conflict monitoring and anterior cingulate cortex: an update. Trends Cogn. Sci. 8, 539-546

43 Desimone, R. and Duncan, J. (1995) Neural mechanisms of selective visual attention. Annu. Rev. Neurosci. 18, 193-222

44 Sander, M.C. et al. (2012) Lifespan age differences in working memory: a two-component framework. Neurosci. Biobehav. Rev. 36, 2007-2033

45 Blanchard-Fields, F. and Hertzog, C. (2000) Age differences in social schematicity. In Generative Mental Processes and Cognitive Resources (von Hecker, U. et al., eds), pp. 175-198, Kluwer Academic

46 Touron, D.R. and Hertzog, C. (2004) Distinguishing age differences in knowledge, strategy use, and confidence during strategic skill acquisition. Psychol. Aging 19, 452-466

47 Freund, A.M. and Baltes, P.B. (2000) The orchestration of selection, optimization, and compensation: an action-theoretical conceptualization of a theory of developmental regulation. In Control of Human Behavior, Mental Processes, and Consciousness (Perrig, W.J. and Grob, A., eds), pp. 35-58, Lawrence Erlbaum Associates

48 Park, D.C. and Reuter-Lorenz, P. (2009) The adaptive brain: aging and neurocognitive scaffolding. Annu. Rev. Psychol. 60, 173-196

49 Touron, D.R. et al. (2007) Moderation of older adults' retrieval reluctance through task instructions and monetary incentives. $J$. Gerontol. B: Psychol. Sci. Soc. Sci. 62, 149-155

50 Spieler, D.H. et al. (2006) Outsourcing cognitive control to the environment: adult age differences in the use of task cues. Psychon. Bull. Rev. 13, 787-793

51 Shing, Y.L. et al. (2012) Memory updating practice across 100 days in the COGITO study. Psychol. Aging 27, 451-461

52 Kong, D. et al. (2012) Functional imaging correlates of impaired distractor suppression following sleep deprivation. Neuroimage 61, $50-55$ 
53 Kool, W. and Botvinick, M.M. (2012) A labor/leisure tradeoff in cognitive control. J. Exp. Psychol. Gen. http://dx.doi.org/10.1037/ a0031048

54 Larson, G.E. et al. (1995) Evaluation of a 'mental effort' hypothesis for correlations between cortical metabolism and intelligence. Intelligence $21,267-278$

55 Burzynska, A. et al. (2013) A scaffold for efficiency in the human brain. J. Neurosci. (in press)

$56 \mathrm{Lu}, \mathrm{H}$. et al. (2011) Alterations in cerebral metabolic rate and blood supply across adult lifespan. Cereb. Cortex 21, 1426-1434

57 Volkow, N.D. et al. (2000) Association between age-related decline in brain dopamine activity and impairment in frontal and cingulate metabolism. Am. J. Psychiatry 157, 75-80

58 Greve, W. and Staudinger, U.M. (2006) Resilience in later adulthood and old age: Resources and potentials for successful aging. In Developmental Psychopathology (2nd ed.) (Ciccetti, D. and Cohen, D.J., eds), pp. 796-840, John Wiley \& Sons

59 Murdock, B.B.J. (1967) Recent developments in short-term memory. Br. J. Psychol. 58, 421-433

60 Craik, F.I.M. and Lockhart, R.S. (1972) Levels of processing: a framework for memory research. J. Verb. Learn. Verb. Behav. 11, 671-684

61 La Voie, D. and Light, L.L. (1994) Adult age differences in repetition priming: a meta-analysis. Psychol. Aging 9, 539-553

62 Luo, L. and Craik, F. (2008) Aging and memory: a cognitive approach. Can. J. Psychiatry 53, 346-353

63 Lachaux, J-P. et al. (1999) Measuring phase synchrony in brain signals. Hum. Brain Mapp. 8, 194-208

64 Lindenberger, U. and Lövdén, M. (2006) Co-constructing human engineering technologies in old age: lifespan psychology as a conceptual foundation. In Lifespan Development and the Brain: The
Perspective of Biocultural Co-constructivism (Baltes, P.B. et al., eds), pp. 350-375, Cambridge University Press

65 Lindenberger, U. et al. (2008) Psychological principles of successful aging technologies: a mini-review. Gerontology 54, 59-68

66 Rogers, W.A. and Fisk, A.D. (2010) Toward a psychological science of advanced technology design for older adults. J. Gerontol. B: Psychol. Sci. Soc. Sci. 65, 645-653

67 Mäntylä, T. (1986) Optimizing cue effectiveness: recall of 500 and 600 incidentally learned words. J. Exp. Psychol. Learn. Mem. Cogn. 12, 66 71

68 Tulving, E. and Thompson, D.M. (1973) Encoding specificity and retrieval processes in episodic memory. Psychol. Rev. 80, 352-373

69 Bäckman, L. and Mäntylä, T. (1988) Effectiveness of self-generated cues in younger and older adults: the role of retentional interval. Int. J. Aging Hum. Dev. 26, 241-249

70 Nehmer, J. et al., eds (2010) Aging and Technology [Special Issue], GeroPsych 23, 53-130

71 Dhami, M.K. et al. (2004) The role of representative design in an ecological approach to cognition. Psychol. Bull. 130, 959-988

72 Lövdén, M. et al. (2010) A theoretical framework for the study of adult cognitive plasticity. Psychol. Bull. 136, 659-676

73 Nyberg, L. et al. (2012) Memory aging and brain maintenance. Trends Cogn. Sci. 16, 292-305

74 Nehmer, J. et al. (2010) Aging and technology: friends, not foes. GeroPsych 23, 55-57

75 Carstensen, L.L. et al. (2006) Aging and the intersection of cognition, motivation and emotion. In Handbook of the Psychology of Aging (6th ed.) (Birren, J. and Schaie, K.W., eds), pp. 343-362, Academic Press

76 Hertzog, C. et al. (2009) Enrichment effects on adult cognitive development: can the functional capacity of older adults be preserved and enhanced? Psychol. Sci. Public Interest 9, 1-65 